\title{
Derivative Expansion of the Effective Action*
}

\author{
Oren Cheyette
}

Ph.D. Thesis

\author{
Laurence Berkeley Laboratory \\ and \\ Department of Physics \\ University of California \\ Berkeley, California
}

'This work was supported by the Director, Office of Energy Research, Office of High Energy and Nuclear Physics, Division of High Energy Physica of the U.S. Department of Energy under Contract DE-AC03-765FO0O98 and in part by the National Science Foundation under grant PHY85-15857. 
DE87 012879

\section{Abstract}

This paper describes some methods for calculating derivative terms in the one loop effective action for a quantum field theory. The functional approach and background field method are first used to derive the general form of the one loop determinant. Then the determinant is expanded in powers of derivatives of the background fields. The form of this expansion is described for the simple case of an interacting scalar field, and then for the more complicated problem of a non-abelian gauge field. Finally, the expansion is applied to the task of calculating Higgs mass dependent effects in the Glashow-Weinberg-Salam model, and all terms which grow with the Higgs mass $M_{H}$ are found in the one loop approximation. The result of this calculation is used to find the dependence of the gauge boson mass ratio $\rho$ on $M_{H}$, and also to estimate the size of corrections to $W$ and $Z$ scattering theorems.

\section{DISCLAIMER}

This report was prepared as an alcount uf work spenmored hy an agency of the Iniled States Government. Neither the llnited States Governnent nor any agency thereof, nor any of their employees, makes anj warratly, express or implict, or assuntes any legal liability or responsibility for the accuracy, completeness, or usefulness of any information, apparatus, prenduct, ar pracess disclosed, or represents that its use would not infringe privitely owned rights Refer. ence herein to any specific cummereial profuct, process, or service by trade name, trademark, manufacturer, or otherwise dies not necessarily constitute or imply its endorsement, recommendation, or favoring by the United States Guvernment or any agency therenf. The views and opinions of authors expressed herein do nut necessarily state or reflect those of the United States Government or any agency thereor. 
This thesis is dedicated to Saba, who got me started on it. 


\section{Acknowledgments}

It is my great pleasure to thank Mary $K$. Gaillard for her tolerant and patient guidance through these last few years. The completion of this research is largely due to the time and effort she devoted to bailing me out of my constant confusions.

I also thank the theory group members with whom I have enjoyed many enlightening discussions: Orlando Alvarez, Bob Cahn, Mahiko Suzuki, Zvi Bern, Pierre Binetruy, Hue-Sun Chan, Mitch Golden, Dae-Sung Hwang, Shobhit Mahajan, Neil Marcus, Jon Yamron, and especially Mike Dugan. 


\section{Contents}

Acknowledgments . . . . . . . . . . . . . . . . ii

I. Introduction ..................... 1

II. The Derivative Expansion . . . . . . . . . . . . . . 6

1. Scalar Field . . . . . . . . . . . . . . . . . 7

2. The Covariant Derivative Expansion . . . . . . . . . 13

111. The Glashow-Weinberg-Salam Model . . . . . . . . . . . . 21

1. The One Loop Calculation . . . . . . . . . . . . 22

2. Phenomenological Consequences ........... 32

IV. Conclusions . . . . . . . . . . . . . . . . 36

References .................... 38 


\section{Introduction}

The action plays a fundamental role in our understanding of both classical and quantum physics. In classical mechanics a field configuration $\phi(x)$ which extremizes the action functional $S[\phi(x)]$ satisfies the equations of motion. The classical action can be quantized through the operator formalism, from which we obtain the familiar Feynman rules of quantum field theory. Another approach to quantizing the action, with perhaps more intuitive appeal than the operator mrthod, is the path integral formalism introduced by Feynman (see, e.g., Quantum Mecharics and Path Integrals by Feynman and Hibbs). la this approach one treats the artion functional as the phase of a complex weight, and sums over all possible field configurations in order to find the expected value of any particular quantity. In the usual treatment, one defines the generating functional for the Green functions of the quantum field theory by

$$
Z[J]=N^{-1} \int \mathcal{D} \phi e^{(i / \hbar)(S[\phi]+J \phi)},
$$

where the integral is taken over all degrees of freedom of the field, and the normalization is defined so that $Z[0]=1$. The generating functional of the connected Green functions is then given by $\frac{i}{\hbar} W[J]=\ln Z[J]$, and the effective action is defined by a Legendre transform,

$$
\Gamma[\bar{\phi}]=W[J]-J \bar{\phi},
$$

where the expectation value of the quantum field $\bar{\phi}$ is an implicit function of the source $J, \bar{\phi}=\frac{\delta W[J]}{\delta J}$. The effective action so defined is the generating functional of the "one particle irreducible" (1PI) Green functions: those whose corresponding Feynman graphs cannot be disconnected by removing an internal line. The effective action is to quantum field theory what the classical action is 
to classical field theory: the exact scattering amplitudes of the theory are given by the tree graphs generated from the appropriate action.

There is an alternative view of the effective action, which makes its relationship to the classical action somewhat more transparent than the description above. In the background feld method, one defines the effective action by including in the classical action the "smearing" effect of quantum fluctuations around a particular background field value. The background field generating functional is defined by

$$
e^{(i / \hbar) W\left[\phi^{D}, J\right]}=N^{-1} \int \mathcal{D} \phi e^{(i / \hbar)\left(S\left[\phi+\phi^{D}\right]+J \phi\right)}
$$

and the background field effective action is given by

$$
\Gamma\left[\phi^{B}, \bar{\phi}\right]=W\left[\phi^{B}, J\right]-J \tilde{\phi},
$$

with $\bar{\phi}=\frac{\delta W\left[\phi^{D}, J\right]}{\delta J}$. It is easy to find the relation between the effective action of the background field method and that defined in equation (1.2) [1]. Shifting the integration variable in (1.3), $\phi \rightarrow \phi-\phi^{B}$, we have

$$
W\left[\phi^{B}, J\right]=W[J]-J \phi^{B} .
$$

We then obtain $\bar{\phi}=\bar{\phi}-\phi^{B}$, where $\bar{\phi}$ is defined as above, and comparing equations (1.4) and $(1.2)$ we find $\Gamma\left[\phi^{B}, \bar{\phi}\right]=\Gamma[\bar{\phi}]$. Setting the expectation of the quantum field $\bar{\phi}$ in the presence of the background field to 0 gives $\Gamma\left[\phi^{D}, 0\right]=\Gamma\left[\phi^{D}\right]$. This tells us that in order to find the effective action we can simply compute the background field effective action for vacuum to vacuum transitions of the quantum field in the presence of a background field $\phi^{B}$. Jackiw [2] showed that the condition $\bar{\phi}=0$ is satisfied by choosing the current $J$ in equation (1.3) to be

$$
J=-\left.\frac{\delta S\left[\phi+\phi^{B}\right]}{\delta \phi}\right|_{\phi=0} .
$$

That is, the expectation of the quantum field $\bar{\phi}$ vanishes for for this choice of $J$. Hence we may wrile

$$
e^{(i / \hbar) \Gamma\left[\phi^{B}\right]}=N^{-1} \int D \phi e^{(i / h)\left(S\left[\phi+\phi^{D}\right]-S^{\prime} \phi\right)},
$$


where $S^{\prime}$ denotes the functional derivative as given in equation (1.6). (From a diagrammatic point of view, this amounts to computing diagrams with no external legs with the quantized action $S\left[\phi+\phi^{B}\right]-S^{\prime} \phi$.) The overall normalization is independent of $\phi^{\text {Ia }}$ (as can be seen from equation (1.1)), so it's just an irrelevant constant term in the effective action, and we won't worry about it from here on. We will also set $\hbar=1$.

The one loop approximation (that is, the approximation of including only tree diagrams and diagrams with one closed loop) is equivalent in the functional approach being used here to the saddle point approximation to the integral in equation (1.7). The loop expansion of (1.7) is found by constructing the functional Taylor series of the exponent, and using the Gaussian integration formula

$$
\int \prod_{i} d x_{i} e^{-\frac{1}{2} x_{m} A_{m m} x_{n}}=C \operatorname{det}^{-\frac{1}{2}} A,
$$

for a positive matrix $A$, where $C$ is a numerical constant. We assume in what follows that after Wick rotating to a Euclidean metric the positivity condition is satisfied, and we absorb the constant $C$ into the normalization $N$. Keeping only the lowest or.'er non-trivial term, we then have

$$
\Gamma\left[\phi^{B}\right]=S\left[\phi^{D}\right]+\left.i \ln \operatorname{det}^{\frac{2}{2}} \frac{\delta^{2} S\left[\phi+\phi^{D}\right]}{\delta \phi^{2}}\right|_{\phi=0} .
$$

This formula, giving the first quantum correction to the classical action, is the object of study in the remainder of this paper.

There are two conventional ways of expanding $\Gamma\left[\phi^{D}\right]$. Viewing $\Gamma\left[\phi^{D}\right]$ as the generating functional of the 1PI Green functions, we may expand the effective action in a functional Taylor series as

$$
\Gamma\left[\phi^{B}\right]=\sum_{n} \frac{1}{n !} \int d x_{1} \cdots d x_{n} \Gamma_{n}\left(x_{1}, \ldots, x_{n}\right) \phi^{B}\left(x_{1}\right) \cdots \phi^{B}\left(x_{n}\right),
$$

where the coefficient functions $\Gamma_{*}$ are the 1PI Green functions (and are non-local in coordinate space). This kind of expansion is the natural result of a diagramunatic calculation, where a Green function for a specific number of external legs 
is calculated. Alternatively, we may find the effective action for configurations near the "coherent" state of a uniform field, writing it as an infinite sum of local terms by expanding it in powers of derivatives of $\phi^{D}(x)$ :

$$
\Gamma\left[\phi^{B}\right]=\int d x\left\{-V\left(\phi^{B}(x)\right)+\frac{1}{2} Z_{2}\left(\phi^{D}(x)\right)\left(\partial \phi^{B}(x)\right)^{2}+\cdots\right\} .
$$

The first term is the effective potential-the effective action for sparetime independent fields. This term is crucial to our understanding of quantum corrections to spontaneous symmetry breaking; the calculation of the effective potential has been widely studied, notably by Coleman and Weinberg[3] and by Jackiw[2]. The remaining terms involve 2 or more derivatives, and until recently little attention had been paid to the problem of their calculation. When it was necessary to evaluate these higher derivative terms, for example in studies of solitons, calculations were generally done by computing some carefully chosen amplitudes by diagrammatic methods, and then reconstructing the effective action [4], or else by complicated functional methods [5]. Over the past two years, though, a number of papers have appeared giving simpler and more elegant methods for constructing the derivative expansion of the effective action in the one loop approximation [6-12]. The second chapter of this paper is concerned with describing some of these methods, developed by this and other authos.

Chapter 2 is divided into two sections. In the first, the derivative expansion is developed for problems involving scalar fields (also applicable to problems with fermions coupled to scalars), and some examples are worked out. The second section describes the covariant derivative expansion, applicable to problems with local or non-linear symmetries, and an example of its application is displayed through the calculation of the QCD $\beta$ function. This calculation, given in fairly explicit detail, should make clear the great computational simplification afforded by this method.

The third chapter concerns the application of the covariant derivative expansion to a more complicated and interesting problem: the gauge sector of 
the Glashow-Weinberg-Salam model of electro-weak interactions. The goal is to understand the dependence of the observed gauge sector (the $W^{ \pm}$and $Z$ weak vecior bosons and the photon) on the characteristic mass scale of the hidden symmetry breaking physics. All we really know at present about that sector is that some effective scalar field has a vacuum expectation value of $250 \mathrm{GeV}$; we know essentially nothing of the mass or other characteristics of the field responsible. If this field is very massive it will be hard to find at accelerators, and if it is composite there may indeed be nothing like the Higgs particle to find-just some heavy mass scale at which some new and currently unknown physics becomes directly observable. We are therefore concerned with extracting whatever low energy tests are possible which depend only on the knowledge that there exist some gauge bosons, and the requirement of unitarity which dictates the exustence of the new physics responsible for the symmetry breaking. Much work in this direction has been done over the last ten years or so [13-18] and will be tested in part by the eagerly awaited results from SLC and LEP. These predictions have been extrarted by using the conventional and often tedious diagrammatic method of computation. That method, applied to problems which possess some special symmetries, has the disadvantage of spoiling those symmetries at intermediate stages of the calculation; they must be recovered at the end, and while that may afford a check on the computations, it leads to unnecessary complexity in the middle. In contrast, the covariant derivative expansion is designed to preserve the symmetries inherent in the problem, and in so doing it simplifies the details of the computation. In chapler 3 the one loop "heavy Higgs" effects will be derived using the covariant derivative expansion, and displayed in the language of the effective action. 


\section{The Derivative Expansion}

In this chapter we will derive some methods of constructing the derivative expansion of the one loop effective action. None of them is completely general in applicability, but together they seem to encompass the various cases of physical iriterest. Methods closely related to these have been studied by a number of authors recently [6-12]. These functional methods have advantages over calculations with Feynman diagrams, in that symmetries of the Lagrangian are preserved at intermediate stages of the calculation; indeed, the existence and explicit preservation of symmetries generally simplifies the calculation, since non-symmetric terms which must later cancel never even appear.

As explained in the introduction, the functional construction of the loop expansion gives the result that the one loop contribution to the effective action is the saddle-point approximation to the functional integral:

$$
S_{1}=\int d^{4} x c_{1}=i \ln \operatorname{det}^{\frac{1}{3}} \frac{\delta^{2} S}{\delta \phi_{i}(x) \delta \phi_{j}(y)}\left[\phi^{B}\right]=\frac{i}{2} \operatorname{Tr} \ln \frac{\delta^{2} S}{\delta \phi_{i}(x) \delta \phi_{j}(y)}\left[\phi^{B}\right]
$$

where $\phi_{i}(x)$ are the fields appearing in the quantum action $S$ ( $i$ denotes the internal indices which may be carried by $\phi$.) The operator

$$
\Delta_{\mathrm{up} i}^{-1}(x, y) \equiv \frac{\delta^{2} S}{\delta \phi_{i}(x) \delta \phi_{j}(y)}\left[\phi^{B}\right]
$$

can be thought of as the inverse of the propagator for the quanturn field $\phi$ in the presence of the background field $\phi^{D}$. In the special case that $\phi^{\mathbb{D}}$ is independent of $x$, the one loop trace may be evaluated easily by Fourier transforming and integrating over momentum, to obtain the result of Coleman and Weinberg for the one loop effective potential [3]. The more general case, of non-constant $\phi^{B}$ can ive isindled by means of the methods to be described telow. 


\section{Scalar Field}

For a renormalizable scalar field theory, the general form of $\Delta_{\text {op }}^{-1}$ is

$$
\Delta_{\mathrm{op} i j}^{-1}(x-y)=-\left[\partial^{2}+U_{i j}\left(\phi^{B}(x)\right)\right] \delta(x-y),
$$

where $U_{i j}\left(\phi^{B}(x)\right)$ is the second derivative of the scalar potential (the non-derivative terms in $S$ ),

$$
U_{i j}\left(\phi^{B}(x)\right)=\frac{\partial^{2} V}{\partial \phi_{i} \partial \phi_{j}}\left(\phi^{B}(x)\right) .
$$

Taking a lead from reference 8 , we split $U_{i j}(x)$ into two pieces: $U_{i j}(x)=$ $U_{i j}\left(x_{0}\right)+U_{1 i j}\left(x_{0}, x\right)$, that is, a constant piece and a coordinate dependent remainder, with $U_{1}\left(x_{0}, x_{0}\right)=0$. Then the one loop trace we are trying to evaluate is

$$
S_{1}=\frac{i}{2} \operatorname{Tr} \ln \left[\left(\partial_{x}^{2} \delta_{i j}+U_{i j}\left(x_{0}\right)+U_{1 i j}\left(x_{0}, x\right)\right) \delta(x-y)\right]
$$

We may split the trace into two pieces (using $\operatorname{Tr} \ln X Y=\operatorname{Tr} \ln X+\operatorname{Tr} \ln Y$, which follows from det $X Y=\operatorname{det} X \operatorname{det} Y$ for matrices $X$ and $Y$ ), and write

$$
S_{1}=\frac{i}{2} \operatorname{Tr} \ln \Delta_{0 u p}^{-1} \delta(x-y)+\frac{i}{2} \operatorname{Tr} \ln \left(1+\Delta_{0 \text { op }} U_{1 i j}\right) \delta(x-y),
$$

where

$$
\Delta_{0 \text { op }}^{-1}=\partial_{x}^{2}+U_{i j}\left(x_{0}\right)
$$

We now Fourier transform to momentum space, and "shift" the momentum by the gradient of $x$. Define operators

$$
A=-i p_{\mu} x^{\mu} \quad \text { and } \quad B=i \partial_{\mu} \frac{\partial}{\partial p_{\mu}}
$$

Then

$$
\operatorname{Tr} \ln \Delta_{\mathrm{op}}^{-1}=\frac{1}{V} \int \frac{d^{4} p}{(2 \pi)^{4}} \operatorname{Tr} \ln e^{B} e^{A} \Delta_{\mathrm{op}}^{-1} e^{-A} e^{-B},
$$

where $V=\delta^{4}(0)$ is the "volume" of the $p$ integral. The factor $\frac{1}{V}$ cancels against a factor of $V$ from the trace on coordinate space (there's a $\delta$ function in $\Delta_{\text {op }}^{-1}$, 
and another one introduced in taking the trace, so one of them comes out as an extra factor $\delta^{4}(0)$ ), and we can drop it from here ou. The trace is invariant under conjugation, and the integral simply averages over all values of $p$ [12]. Using the relations

$$
e^{X} Y e^{-X}=e^{L_{x} Y} \quad \text { where } \quad L_{X} Y \equiv[X, Y]
$$

and

$$
L_{A} \partial_{\mu}=i p_{\mu}, \quad L_{B} p_{\mu}=i \partial_{\mu} \quad \text { and } \quad L_{B} U_{1}=i\left(\partial_{\mu} U\right) \frac{\partial}{\partial p_{\mu}},
$$

we may rewrite equation $(2.5)$ as $[10,12]$

$$
S_{1}=\int \frac{d^{4} p}{(2 \pi)^{4}} \operatorname{Tr}\left(\ln \Delta^{-1}+\ln (1+\Delta \widehat{U})\right) .
$$

where

$$
\Delta^{-1}=-p^{2}+U\left(x_{0}\right) \quad \text { and } \quad \widehat{U}=\sum_{n=0}^{\infty} \frac{1}{n !}\left(\partial_{\mu_{n}} \cdots \partial_{\mu_{n}} U_{1}\left(x_{0}, x\right)\right) \frac{i^{n} \partial^{n}}{\partial p_{\mu_{1}} \cdots \partial p_{\mu_{n}}}
$$

The first term in this expression is the effective potential; the second piece gives all terms involving derivatives of the background field. We can now expand the logarithm, integrate over the momentum $p$, and find the derivative terms oui is any desired order.

There is one point in this process that requires some explanation. The expansion for $\widehat{U}$ starts with the $n=0$ term $U_{1}(x)$, so it might appear that one needs to keep arbitrarily high order terms in the Taylor expansion of the logarithm to find, say, the two derivative terms. Fortunately, this is not the case: if we are interested in terms with up to $N$ derivatives, we need only retain terms in the expansion of equation (2.10) with up to $N$ powers of $\widehat{U}$. The reason is as follows. The effective action of equation (2.10) is independent of $x_{0}$-even though $x_{0}$ appears in $\Delta$ and $U_{1}$, this is just an artifact of the splitting introduced in equation (2.4). The form of the expansion of the second logarithm in equation 
(2.10) after the momentum integration and with the space integration in the trace made explicit, is a sum of terms of the form (schematically)

$$
\int d x \operatorname{tr} \frac{\left(\partial_{x} U(x)\right)^{k}}{U\left(x_{0}\right)^{m}}\left(U(x)-U\left(x_{0}\right)\right)^{n}
$$

The denominator may be written as $U(x)-\left(U(x)-U\left(x_{0}\right)\right)$, so this can be reexpanded in terms of expressions of the form

$$
\int d x \operatorname{tr} \frac{\left(\partial_{x} U(x)\right)^{k}}{U(x)^{m}}\left(\left(U(x)-U\left(x_{0}\right)\right)^{n} .\right.
$$

(Here tr signifies the trace over internal indices only.) The only term of this sort which is independent of $x_{0}$ is one with $n=0$; hence the terms with $n \geq 1$ must all cancel out. The key point he. is that $\partial_{x} U_{1}\left(x_{0}, x\right)=\partial_{x} U(x)$, which is independent of $x_{0} \rightarrow$ so a term survives as long as it has enough derivatives to eliminate all the dependence on $x_{0}$. There is also the effective potential term (from the first logarithm), which may be added to the terms with $k=0$ in the expansion of the second term to give the effective potential at $x$. We thus have an expansion in numbers of derivatives of an effective action which can be written as

$$
S_{1}\left[\phi^{B}\right]=\int d x \mathcal{L}_{1}\left(\phi^{B}(x)\right)
$$

It is convenient sometimes to introduce a slight simplification in the derivation given above. Using the relation

$$
\operatorname{Tr} \ln (A+B)=\operatorname{Tr} \ln A+\int_{0}^{1} d z \operatorname{Tr} \frac{1}{A+z B} B
$$

we may rewrite the one loop contribution (2.4) as

$$
\begin{aligned}
S_{1}=\frac{i}{2} \int \frac{d^{4} p}{(2 \pi)^{4}} & \operatorname{Tr} \ln \Delta^{-1}+\frac{i}{2} \int_{0}^{1} d z \int \frac{d^{4} p}{(2 \pi)^{4}} \\
& \operatorname{Tr} \sum_{n=0}^{\infty}(-z)^{n}\left[\Delta_{1} \sum_{m=1}^{\infty} \frac{1}{m !}\left(\partial_{\mu_{1}} \cdots \partial_{\mu_{m}} U_{1}\right) \frac{z^{m} \partial^{m}}{\partial p_{\mu_{1}} \cdots \partial p_{\mu_{m}}}\right]^{n} \Delta_{1} U_{1},
\end{aligned}
$$


with

$$
\Delta_{1}(z)=\frac{1}{-p^{2}+U\left(x_{0}\right)+z U_{1}(x)}
$$

The $n=0$ term in the outer sum may be absorbed into the effective potential term, which then becomes the effective potential at $x$ instead of at $z_{0}$. This may not look simpler than the expansion of equation (2.10), but in fact there are fewer terms to deal with, and some of the combinatorics of the expansion are postponed to a late stage of the calculation, where they return as ccefficients in the expansion in powers of $U_{1}$.

As a first example consider the simplest case, that of a single cumponent scalar field, described by the Lagrangian

$$
\mathcal{L}=\frac{1}{2}\left(\partial_{\mu} \phi^{2}\right)-V(\phi) \quad \text { where } \quad V(\phi)=\frac{1}{2} m_{1}^{2} \phi^{2}+\frac{1}{3 !} m_{2} \phi^{3}+\frac{1}{4 !} \lambda \phi^{4} .
$$

In this case we have

$$
U=m_{1}^{2}+m_{2} \phi+\frac{\lambda}{2} \phi^{2}
$$

there are no matrices to worry about, and equation (2.16) may be expanded to give

$$
\begin{aligned}
& \mathcal{L}_{1}=\frac{i}{2} \int_{0}^{1} d z \int \frac{d^{4} p}{(2 \pi)^{4}} \Delta_{1} U_{1}\left\{\frac{z}{2} \frac{\partial^{2}}{\partial p_{\mu} \partial p_{\nu}} \Delta_{1} \partial_{\mu} \partial_{\nu} U\right. \\
&-\frac{z}{4 !} \partial_{\mu} \partial_{\nu} \partial_{\rho} \partial_{\sigma} U \frac{\partial^{4}}{\partial p_{\mu} \partial p_{\nu} \partial p_{\rho} \partial p_{\sigma}} \Delta_{1} \\
&+\frac{z^{2}}{3 !} \partial_{\mu} U \partial_{\nu} \partial_{\rho} \partial_{\sigma} U\left[\frac{\partial}{\partial p_{\mu}} \Delta_{1} \frac{\partial^{3}}{\partial p_{\nu} \partial p_{\rho} \partial p_{\sigma}} \Delta_{1}+\frac{\partial^{3}}{\partial p_{\nu} \partial p_{\rho} \partial p_{\sigma}} \Delta_{1} \frac{\partial}{\partial p_{\mu}} \Delta_{1}\right] \\
&+\frac{z^{2}}{(2 !)^{2}} \partial_{\mu} \partial_{\nu} U \partial_{\rho} \delta_{\sigma} U \frac{\partial^{2}}{\partial p_{\mu} \partial p_{\nu}} \Delta_{1} \frac{\partial^{2}}{\partial p_{\rho} \partial p_{\sigma}} \Delta_{1} \\
&-\frac{z^{3}}{2 !} \partial_{\mu} U \partial_{\nu} U \partial_{\mu} \partial_{\sigma} U\left[\frac{\partial}{\partial p_{\mu}} \Delta_{1} \frac{\partial}{\partial p_{\nu}} \Delta_{1} \frac{\partial^{2}}{\partial p_{\rho} \partial p_{\sigma}} \Delta_{1}\right. \\
&\left.\left.+\frac{\partial^{2}}{\partial p_{\rho} \partial p_{\sigma}} \Delta_{1} \frac{\partial}{\partial p_{\mu}} \Delta_{1} \frac{\partial}{\partial p_{\nu}} \Delta_{1}+\frac{\partial}{\partial p_{\nu}} \Delta_{1} \frac{\partial^{2}}{\partial p_{\rho} \partial p_{\sigma}} \Delta_{1} \frac{\partial}{\partial p_{\rho}} \Delta_{1}\right]\right\} \\
&+ \text { effective potential + higher derivatives. }
\end{aligned}
$$


The momentum derivatives and integrals may be evaluated using the formulas

$$
\begin{aligned}
& \frac{\partial}{\partial p_{\mu}} \Delta_{1}=2 p^{\mu} \Delta_{1}^{2}, \quad \frac{\partial^{2}}{\partial p_{\mu} \partial p_{\nu}} \Delta_{1}=2 \Delta_{1}^{2}\left(g^{\mu \nu}+4 p^{\mu} p^{\nu} \Delta_{1}\right) \\
& \int \frac{d^{4} p}{(2 \pi)^{4}} p^{2 s} \Delta_{1}^{n}=(-1)^{s} i\left(U\left(x_{0}\right)+z U_{1}\left(x_{0}, x\right)\right)^{-(n-s-2)} \frac{\Gamma(s+2) \Gamma(n-s-2)}{-16 \pi^{2} \Gamma(n)}
\end{aligned}
$$

Finally, we may expand in powers of $U_{1}\left(x_{0}, x\right)$, retaining as many powers as there are derivatives in the subexpression, and do the $z$ integration. The result is

$$
\mathcal{C}_{1}=\frac{1}{64 \pi^{2}}\left\{\frac{1}{6} \frac{\left(\partial_{\mu} U\right)^{2}}{U}+\frac{1}{60} \frac{\left(\partial^{2} U\right)^{2}}{U^{2}}-\frac{1}{45} \frac{\partial^{2} U\left(\partial_{\mu} U\right)^{2}}{U^{3}}+\frac{1}{120} \frac{\left(\partial_{\mu} U\right)^{4}}{U^{4}}\right\}
$$

+ effective potential + higher derivatives,

in agreement with Fraser[7], who used a somewhat different method, and also in agreement with Chan[6], except for a sign error in one term of his published result. (The results may be compared by adding a total divergence to equation (2.22) to cast it into the appropriate form.)

The case of a scalar field theory with internal symmetry introduces the complication of matrix valued $U$. As an example, we compute the two derivative terms for a scalar theory with global $O(N)$ symmetry. (A calculation of the two and four derivative terms for this model was published at about the same time as this one by Aitchison and Fraser [8], and somewhat later also by Chan [12]. The various results are all in agreement.) The model Lagrangian is

$$
\mathcal{L}=\frac{1}{2}\left(\partial_{\mu} \phi\right)^{2}-\frac{1}{2} m^{2} \phi^{2}-\frac{1}{4 !} \lambda\left(\phi^{2}\right)^{2}
$$

from which we find

$$
\Delta_{\text {op } i j}^{-1}(x-y)=\left[\left(-\partial^{2}-U_{L}(x)\right) Q_{L i j}(x)+\left(-\partial^{2}-U_{T}(x)\right) Q_{T i j}(x)\right] \delta(x-y),
$$


where the longitudinal and transverse mass and projections are given by

$$
\begin{aligned}
U_{L} & =m^{2}+\frac{1}{2} \lambda \phi^{2}, & U_{T} & =m^{2}+\frac{1}{6} \lambda \phi^{2} \\
Q_{L_{i j}} & =\frac{\phi_{i} \phi_{j}}{\phi^{2}}, & Q_{T i j} & =\delta_{i j}-\frac{\phi_{i} \phi_{j}}{\phi^{i}}
\end{aligned}
$$

and we have the matrir relation for the projections

$$
Q_{a} Q_{b}=\delta_{n b} Q_{a} \quad \text { where } \quad(a, b)=(R, L)
$$

Again, we split $\Delta_{\text {op }}^{-1}$ into an $x$ independent piece

$$
\Delta_{0, p_{p}}^{-1}=\left[\left(-\partial^{2}-U_{L}\left(x_{0}\right)\right) Q_{L}\left(x_{0}\right)+\left(-\partial^{2}-U_{T}\left(x_{0}\right)\right) Q_{T}\left(x_{0}\right)\right]
$$

and the remainder

$$
U_{1}=\left(U_{L}(x) Q_{L}(x)+U_{T}(x) Q_{T}(x)\right)-\left(U_{L}\left(x_{0}\right) Q_{L}\left(x_{0}\right)+U_{T}\left(x_{0}\right) Q_{T}\left(x_{0}\right)\right)
$$

Then equation (2.16) may be written out as

$$
\begin{aligned}
\mathcal{L}_{1}= & \frac{i}{2} \int \frac{d^{4} p}{(2 \pi)^{4}}\left\{\operatorname{tr} \ln \Delta_{L}^{-1}+(N-1) \operatorname{tr} \ln \Delta_{T}^{-1}\right\} \\
+ & \frac{i}{2} \int_{0}^{1} d z \int \frac{d^{4} p}{(2 \pi)^{4}} \operatorname{tr} \sum_{n=0}^{\infty}(-z)^{n}\left[\left(\Delta_{L} Q_{L}\left(x_{0}\right)+\Delta_{T} Q_{T}\left(x_{0}\right)\right) \widehat{U}^{\prime}\right]^{n} \\
& {\left[\Delta_{L} Q_{L}\left(x_{0}\right)+\Delta_{T} Q_{T}\left(x_{0}\right)\right]\left[U_{L 1}(x) Q_{L}\left(x_{0}\right)+U_{T 1}(x) Q_{T}\left(x_{0}\right)\right.} \\
& \left.+\left(U_{L}(x)-U_{T}(x)\right) Q_{L 1}(x)\right]
\end{aligned}
$$

where " $\mathrm{tr}^{n}$ denotes the $\mathrm{O}(N)$ trace, and the terms in this equation are given by

$$
\Delta_{a}=\frac{1}{-p^{2}+U_{a}\left(x_{0}\right)+z U_{a 1}(x)}, \quad U_{a 1}=U_{a}(x)-U_{a}\left(x_{0}\right),
$$

and

$$
\begin{aligned}
& \hat{U}^{\prime}=\left(U_{L}(x)-U_{T}(x)\right)\left(Q_{L}(x)-Q_{L}\left(x_{0}\right)\right)+\sum_{m=1}^{\infty} \frac{1}{m !} \partial_{\mu_{1}} \cdots \partial_{\mu_{m}} \\
& {\left[U_{L_{1}}(x) Q_{L}\left(x_{0}\right)\right.}+U_{T 1}(x) Q_{T}\left(x_{0}\right) \\
&\left.+\left(U_{L}(x)-U_{T}(x)\right)\left(Q_{L}(x)-Q_{L}\left(x_{0}\right)\right)\right] \frac{i^{m} \partial^{m}}{\partial p_{\mu_{1}} \cdots \partial p_{\mu_{m}}}
\end{aligned}
$$


The projections $Q_{L}$ and $Q_{T}$ permit us to split the effective Lagrangian into three parts-a part due solely to longitudinal modes, one from the transverse modes, and a mixed part whose terms involve the change in the projection, $Q_{L}(x)-Q_{L}\left(x_{0}\right):$

$$
\mathcal{L}_{1}=\mathcal{L}_{1}\left[U_{L}\right]+(N-1) \mathcal{L}_{1}\left[U_{T}\right]+\text { mixed terms }
$$

where $\mathcal{L}_{1}\left[U_{\mathrm{a}}\right]$ is the result of equation (2.22) with $U_{\mathrm{a}}$ substituted for $U$, and the mixed terms are what's left over (and subleading in $N$ ). We easily obtain the two derivative terms,

$$
\begin{aligned}
\mathcal{L}_{1}= & \frac{1}{1 \delta \pi^{2}}\left\{\frac{\lambda^{2}}{24}\left(\phi_{i} \partial_{\mu} \phi^{i}\right)^{2}\left[\frac{1}{U_{L}}+\frac{N-1}{9} \frac{1}{U_{T}}\right]\right. \\
& \left.-\frac{1}{2}\left(\frac{\left(\partial_{\mu} \phi_{i}\right)^{\prime}}{\phi^{2}}-\frac{\left(\phi_{i} \partial_{\mu} \phi^{i}\right)^{2}}{\phi^{4}}\right)\left[\frac{U_{L} U_{T}}{\left\{U_{L}-U_{T}\right)} \ln \frac{U_{L}}{U_{T}}-\frac{U_{L}+U_{T}}{2}\right]\right\}
\end{aligned}
$$

+ effective potential + higher derivatives .

It's interesting to see that the leading behavior for large $N$ is just given by the expression for a single scalar field, since equation (2.31) is then dominated by $N \mathcal{C}_{1}\left[U_{T}\right]$.

There is no obstacle to extending this teclunique beyond one loop order in perturbation theory. The functional expansion of Jackiw [2] tells us that to do this we just write down the connected one-particle irreducible diagrams generated by the Lagrangian in the presence of the background field $\phi^{B}$. In this case we must first express the diagrams in coordinate space, then make as many transformations of the form given in (2.7) as there are propagators. The sesult then has the form of integrals over the loop momenta of the trace of a product of propagators of the form $\left[-p^{2}+U\left(x_{0}\right)+\widehat{U}\left(x_{0}, x\right)\right]^{-1}$, which may be expanded in powers of derivatives.

\section{The Covariant Derivative Expansion}

The method described in section 1 for handling scalar fields is useful only 
for those problems where the derivative operator has no spacetime dependence (or when that dependence may be transformed away, e.g., by a gauge transformation). For example, the method described above may be applied to problems involving fermious coupled to scalars, for which the derivative multiplies a constant matrix; however, it is not useful for problems involving non-trivial gauge fields. A more general expansion, applicable to this case has been given in reference 10; its derivation is sketched below.

The generic expression we seek to evaluate is $\operatorname{Tr} \ln \left(d^{2}+M^{2}\right)$. Define operators, like those of equation (2.7): $A=-i p_{\mu} x^{\mu}$ and $B=i \dot{a}_{\mu} \frac{\partial}{\partial p_{\mu}}$. Then

$$
\operatorname{Tr} \ln \left(d^{2}+M^{2}\right)=\frac{1}{V} \int \frac{d^{A} p}{(2 \pi)^{4}} \operatorname{Tr} \ln e^{B} e^{A}\left(d^{2}+M^{2}\right) e^{-A} e^{-B}
$$

as before. Using

$$
L_{A} d_{\mu}=i p_{\mu}, \quad L_{B} d_{\mu}=i\left[d_{\nu}, d_{\mu}\right] \frac{\partial}{\partial p_{\nu}} \quad \text { and } \quad L_{B} p_{\mu}=i d_{\mu}
$$

we may rewrite the trace as

$$
\operatorname{Tr} \ln \left(d^{2}+M^{2}\right)=\int \frac{d^{4} p}{(2 \pi)^{4}} \operatorname{Tr} \ln \left(\left(i p_{\mu}+i \tilde{G}_{\nu \mu} \frac{\partial}{\partial p_{\nu}}\right)^{2}+M^{2}+\widehat{\delta M^{2}}\right),
$$

where

$$
\widehat{\delta M^{2}}=\sum_{n=1}^{\infty} \frac{1}{n !}\left[d_{\mu_{1}}, \cdots\left[d_{\mu_{n}}, M^{2}\right] \cdots\right] \frac{i^{\prime \prime} \partial^{n}}{\partial p_{\mu_{1}} \cdots \partial p_{\mu_{n}}}
$$

and

$$
\tilde{G}_{\nu \mu}=\sum_{n=0}^{\infty} \frac{(n+1)}{(n+2) !}\left[d_{\mu_{1}}, \cdots\left[d_{\mu_{n}},\left[d_{\nu}, d_{\mu}\right]\right] \cdots\right] \frac{i^{n} \partial^{n}}{\partial p_{\mu_{1}} \cdots \partial p_{\mu_{n}}}
$$

Note that the trace here is not a trace in momentum space, so that the usual operations that may be performed on the trace of the logarithm (such as Tr In $X Y=$ $\operatorname{Tr} \ln X+\operatorname{Tr} \ln Y$ ) cannot be used.

The expansion of the righthand side of equation (2.35) has been done in various ways. Chan[12] makes only the first transformation, involving $A$, then since 
$p$ commutes with everything the logarithm can be expanded and the result rearranged so that $d_{\beta \alpha}$ only appears in commutators. In this approach the covariance of the final result is not manifest at intermediate stages of the calculation-it is not apparent that one can rearrange terms so that $d_{\mu}$ only appears in commutators. The gauge invariance of the trace guarantees that this can be done, but the manner in which the cancellations must occur is not easily seell. This method is essentially the same as that of Aitchison and Fraser[7,8], extended to the case of covariant derivatives.

Alternatively, Gaillard[10] makes the observation that the one loop expression has to be regulated--the integrand grows at large momenta-so she uses a subtraction procedure which simultaneously regulates the integral and makes the expansion tractable. (In effect, the subtraction introduces into the calculation the counterterms necessary to cancel the infinities before the momentum integrals are evaluated). While subtractions may come into conflict with symmetry principles in ordinary diagrammatic calculations, in this case they do not, because the regulated action is manifestly invariant with respect to the background field symmetry transformations at each siep. It is only syınmetries associated with fields which are integrated over in the functional integral which are affected, and those fields aren't present in the final answer. This regularization is especially useful in studying non-reuormalizable theories, for which the subtraction point replaces the mass scale at which real pliysics intervenes to cut of the divergences. On occasion, though, this method has proved slightlyr inconvenient (as in the calculation of chapter 3), so what follows is yei another expansion procedure, with its own advantages and problems.

Let $\Delta^{-1}=-p^{2}+M^{2}$, and let $\widehat{U}$ be what's left over in the expression in parentheses of equation (2.35):

$$
\widehat{U}=\widehat{\delta M^{2}}-\left\{p^{\mu}, \tilde{G}_{\nu \mu}\right\} \frac{\partial}{\partial p_{\nu}}-\tilde{G}_{\nu \mu} \tilde{G}_{\rho}^{\mu} \frac{\partial^{2}}{\partial p_{\nu} \partial p_{\rho}} .
$$

Note that every term in $\widehat{U}$ involves at least one power of $\frac{\partial}{\partial p}$, so that any term 
with $\widehat{U}$ sitting all the way to the right vanishes. Using the Baker-Hausdorff formula $e^{A} \mathrm{e}^{B}=e^{f(A, B)}$, where $H(A, B)=A+B+\frac{1}{2}[A, B]+\frac{1}{12}[A,[A, B]]+\frac{1}{12}[B,[B, A]]-\frac{1}{24}[A,[B,[A, B]]]+\cdots$

the righthand side of equation (2.35) may be expanded to give

$$
\begin{aligned}
\operatorname{Tr} \ln \left(d^{2}+M^{2}\right)=\int \frac{d^{4} p}{(2 \pi)^{4}} & \operatorname{Tr} \ln \left(\Delta^{-1}+\widehat{U}\right) \\
=\int \frac{d^{4} p}{(2 \pi)^{4}} & \operatorname{Tr}\left(\ln \Delta^{-1}+\ln (1+\Delta \widehat{U})+\frac{1}{2}\left[\ln \Delta^{-1}, \ln (1+\Delta \widehat{U})\right]\right. \\
& +\frac{1}{12}\left[\ln \Delta^{-1},\left[\ln \Delta^{-1}, \ln (1+\Delta \widehat{U})\right]\right] \\
& \left.+\frac{1}{12}\left[\ln (1+\Delta \widehat{U}),\left[\ln (1+\Delta \widehat{U}), \ln \Delta^{-1}\right]\right]\right)+\cdots
\end{aligned}
$$

The second term on the righthand side may be dropped because it always has a $\widehat{U}$ to the right. By using the identity

$$
\left[\ln X_{i} Y\right]=\sum_{n=1}^{\infty} \frac{1}{n} X^{-n} L_{X}^{n} Y
$$

(which may be proved by parametric differentiation), and expanding $\ln (1+$ $\Delta \widehat{U}$ ), each commutator may be written in terms of commutators of $\Delta^{-1}$ and $\tilde{U}$. In general, this series does not generate precisely an expansion in powers of covariant derivatives. Rather, it is a simultaneous expansion in derivatives and commutators of $M^{2}$ with derivatives. Thus, using equation (2.40) with terms including up to 4 commutators gives all terms with 4 covariant derivatives, or 3 derivatives and one commutator with $M^{2}$, and so on. However, this expansion is still useful for finding terms with some number of $d$ rivatives and a particular degree of convergence (or divergence), because each commutator increases the degree of convergence of the expression by one or more powers of $p^{-1}$. This may be seen by simple power counting: each commutator generates either a derivative or a cormmutator with a matrix of dimension $\geq 2$ in the final result. Since the effective Lagrangian has dimension 4, there must be at least as many powers of $p^{-1}$ in the integrand as there are commutators. 
By way of illustration of the uses of the covariant derivative expansion, we will calculate the one-loop Yang-Mills $\beta$ function. Many parts of this calculation will be directly applicabie to the more complicated problem of finding the effective action expansion for the Glashow-Weinberg-Salam model.

The pure Yang-Mills Lagrangian for a simple Lie group may be written

$$
\mathcal{C}=-\frac{1}{4 S} \operatorname{tr} F_{\mu \nu} F^{\mu \mu^{\prime \prime}}
$$

$S$ is defined by $f^{a b x} f^{a b x i}=S \delta^{c d}$ and is equal to $N$ for $\operatorname{SU}(N)$. $f^{a b c}$ are the structure constants of the algebra, and $F_{\mu \nu}$ is the field strength defined by

$$
F_{\mu \nu}=\frac{i}{g}\left[D_{\mu,}, D_{\nu}\right] \quad \text { where } \quad D_{\mu}^{a b}=\delta^{a b} \partial_{\mu}-g f^{a b c}\left(A_{\beta, c}+Q_{\mu c}\right) .
$$

The gauge field has been split into a background part $A$ and a quant' 'n part $Q$. Writing

$$
D_{\mu}^{a b}=\bar{D}_{\mu}^{a b}-g f^{a b c} Q_{\mu c} \quad \text { and } \quad \bar{F}_{\mu \nu}=\frac{i}{g}\left[\bar{D}_{\mu}, \bar{D}_{\nu}\right]
$$

one may then express the terms of order $Q^{2}$ in the quantum action as

$$
\frac{1}{2} Q_{\mu}\left(\bar{D}^{2} g^{\prime \mu}-\bar{D}^{\mu} \bar{D}^{\nu}-2 i g \bar{F}^{\prime \mu}\right) Q_{\nu} \equiv \frac{1}{2} Q^{\mu} \Delta_{\mu \nu}^{-1} Q^{\nu}
$$

In order to perform the functional integral, the Lagrangian nust be gauge fixed, and it is convenient for our purposes to chuose a background-field covariant Iandau gauge: $\bar{D}_{\mu} Q^{\mu}=0$. We implement this by putting a delta function $\delta\left(\bar{D}_{\mu} Q^{\prime \prime}\right)$ into the functional integral, and then exponentiating it,

$$
\delta\left(\bar{D}_{\mu} Q^{\mu}\right)=\int \mathcal{D} \alpha e^{i \int d x \alpha B_{\mu} Q^{\mu}}
$$

so that the Lagrangian becomes

$$
\mathcal{C} \rightarrow \mathcal{L}+i \alpha \bar{D}_{\mu} Q^{\mu}
$$

We then rewrite the resulting expression to eliminate the $Q-a$ cross term $" y$ shifting $Q$ :

$$
Q_{\mu} \rightarrow Q_{\mu}+i \Delta_{\mu} \bar{D}^{\nu} \alpha
$$


Then the bilinear term in the Lagrangian becomes

$$
\frac{1}{2} Q^{\mu} \Delta_{\mu \nu}^{-1} Q^{\mu}-\frac{1}{2} \alpha \bar{D}^{\mu} \Delta_{\mu \nu} \bar{D}^{\mu} \alpha
$$

The gauge condition allows us to simplify the kinetic operator $\Delta_{\mu \mu}^{-1}$ by the replacement

$$
\Delta_{\mu \nu}^{-1} \rightarrow \bar{D}^{2} g_{l \mu}-2 i g \bar{F}_{\mu \mu}
$$

The compensating ghost factor at one loop is just $-i \ln \operatorname{det} \bar{D}^{2}$.

Putting these pieces logether, we find that the one loop effective action is given by

$$
S_{1}=\frac{i}{2} \operatorname{Tr} \ln \Delta_{\mu \nu}^{-1}+\frac{i}{2} \operatorname{Tr} \ln \bar{D}^{\mu} \Delta_{\mu \omega} \bar{D}^{\mu}-i \operatorname{Tr} \ln \bar{D}^{2}
$$

where the terms are due to gauge, auxiliary, and ghost field loops respectively.

It turns out that the auxiliary field term vanishes. This can be seen by gauge fixing instead to Feynman gauge, adding a gauge fixing term $-\frac{1}{2}\left(\bar{D}_{\mu} Q^{\mu}\right)^{2}$ to the action. This cancels the $\bar{D}^{\mu} \bar{D}^{\mu}$ term in $\Delta_{\mu \nu}^{-1}$, giving the same kinetic operator as in (2.50). The ghost determinant is also exactly the same at one loop; however, with this method of gauge fixing, there's no awxiliary field term. So the second term given above in $S_{1}$ must vanish, by gauge invariance.

The gauge field term may be expanded as

$$
\frac{i}{2} \operatorname{Tr} \ln \Delta_{\mu \omega}^{-1}=2 i \operatorname{Tr} \ln \bar{D}^{2}+\frac{i}{2} \operatorname{Tr} \ln \left(g_{\mu \omega}-2 i g \bar{F}_{i \omega} \frac{1}{\bar{D}^{2}}\right) .
$$

The ghost cancels half of the first of these two terms, leaving for the one loop effective action

$$
S_{1}=i \operatorname{Tr} \ln \bar{D}^{2}+\frac{i}{2} \operatorname{Tr} \ln \left(g_{\mu \nu}-2 i g \bar{F}_{\mu \omega} \frac{i}{\bar{D}^{2}}\right) .
$$

The second term may be expanded by using the conjugations (2.3/), as in the derivation of equation (2.35), to give an expansion in covariant derivatives, and the expansion of the first term is given by equation (2.40). Since we are only interested in the logarithmically divergent part, only a few terms need to be 
kept. It is convenient to add an infrared cutoff mass (just to avoid the issue of infrared problems). Then we have

$$
\begin{aligned}
i \operatorname{Tr} \ln \left(\bar{D}^{2}+m^{2}\right)=i \int \frac{d^{4} p}{(2 \pi)^{4}} & \operatorname{Tr}\left\{\ln \Delta^{-1}+\frac{1}{2}\left[\ln \Delta^{-1}, \Delta \widehat{U}\right]\right. \\
& \left.+\frac{1}{12}\left[\ln \Delta^{-1},\left[\ln \Delta^{-1}, \Delta \bar{U}\right]\right]\right\}+ \text { finite terms }
\end{aligned}
$$

where $\Delta^{-1}=-p^{2}+m^{2}$. The first term is field independent, so we can drop it. Using equation (2.41), the log divergent part may be rewritten as

$i \operatorname{Tr} \ln \left(\bar{D}^{2}+m^{2}\right)=i \int \frac{d^{4} p}{(2 \pi)^{4}} \operatorname{Tr}\left\{\frac{1}{2} \Delta^{2}\left[\Delta^{-1}, \widehat{U}\right]+\frac{1}{3} \Delta^{3}\left[\Delta^{-1},\left[\Delta^{-1}, \widehat{U}\right]\right]\right\}+\cdots$

Using the antisymmetry of $\bar{F}_{\mu \omega}$ and the Jacobi identity, we find that the part of $\widehat{U}$ involving $\left\{p^{\mu}, \bar{F}_{\mu \nu}\right\} \frac{\partial}{\partial \rho_{\nu}}$ doesn't contribute to this order. The remaining relevant part of $\widehat{U}$ is then just $\frac{g^{2}}{4} \bar{F}_{j \omega} \bar{F}^{\mu}{ }_{\rho} \frac{\partial^{2}}{\partial p_{\rho} \partial p_{\nu}}$, and we have

$$
\left[\Delta^{-1}, \widehat{U}\right] \rightarrow \frac{g^{2}}{2} \bar{F}_{\mu \omega} \bar{F}^{\prime \mu \nu}
$$

and

$$
\left[\Delta^{-1},\left[\Delta^{-1}, \bar{U}\right]\right] \rightarrow 2 g^{2} p^{\nu} p^{\rho} \bar{F}_{\mu} \bar{F}_{\rho}^{\mu} .
$$

Finally, then, we find the field-dependent log divergence to be

$$
i \frac{g^{2}}{12} \int \frac{d^{4} p}{(2 \pi)^{4}} \operatorname{Tr}\left\{3 \Delta^{2} \bar{F}_{\mu \nu} \bar{F}^{\mu \nu}+8 \Delta^{3} p^{\nu} p^{\mu} \bar{F}_{\mu \nu} \bar{F}_{\rho}^{\mu}\right\} \text {. }
$$

Using dimensional regularization in $4-2 \epsilon$ dimensions, the divergent part is given by

$$
-\frac{1}{\epsilon} \cdot \frac{1}{16 \pi^{2}} \cdot \frac{1}{12} g^{2} \mu^{-2 \epsilon} \cdot \operatorname{Tr} \bar{F}_{\mu \nu} \bar{F}^{\prime \omega}
$$

where $\mu$ is the regularization mass scale.

The remaining divergent term in $S_{1}$, coming from the second trace in equation (2.53), is

$$
i g^{2} \int \frac{d^{4} p}{(2 \pi)^{4}} \operatorname{Tr} \bar{F}_{\mu \nu} \bar{F}^{\prime \prime \prime} \Delta^{2}=\frac{1}{\epsilon} \cdot \frac{1}{16 \pi^{2}} g^{2} \mu^{-2 \epsilon} \operatorname{Tr} \bar{F}_{\mu \nu} \bar{F}^{\prime \mu \nu} .
$$


Adding this to $(2.59)$, we find

$$
\mathcal{L}_{1}=\frac{1}{\epsilon} \cdot \frac{1}{16 \pi^{2}} \cdot \frac{11}{12} g^{2} \mu^{-2 \epsilon} \operatorname{tr} \bar{F}_{14} \bar{F}^{\prime \nu}+\text { finite } .
$$

To renormalize the theory, we subtract this as a counterterm in the original Lagrangian, which may then be written as

$$
\mathcal{C}_{R}=-\frac{1}{4 S} Z_{A}^{2} \text { tr } F_{1 \mu} F^{\mu \nu} \quad \text { with } \quad Z_{A}^{2}=1+\frac{1}{\epsilon} \cdot \frac{11}{48 \pi^{2}} S g^{2} \mu^{-2 \varepsilon} .
$$

From this expression we can compute the $\beta$ function, and find

$$
\beta(g)=\frac{\partial g(\mu)}{\partial \ln \mu}=-\frac{11}{48 \pi^{2}} S_{g^{3}}^{3},
$$

as expected. Note that only the one rencrmalization constant $Z_{A}$ was required in this case, and that it is gauge independent (because the background field was never gauge fixed), as opposed to the usual situation with two gauge dependent renormalization constants which must be combined to obtain the gauge invariant result (2.63). 


\section{The Glashow-Weinberg-Salam Model}

We can now address the problem of calculating the effective derivative interactions in the Glashow-Weinberg-Salam (GWS) model. We will consider the model in the limit where the physical Higgs mass is large compared to other scales, and neglect fermions. Our aim is to understand the consequences for "lowenergy" physics of including in the theory only the spectrum of particles which we already know exists around the symmetry breaking scale of the electro-weak interactions, i.e., the transverse gauge bosons and the Goldstone scalars which give them their mass. A theory with only these fields is a non-renormalizable one: a gauged nou-linear $\sigma$ model or, equivalently, a massive Yang-Mills theory (in this case, the Glashow model). In this picture the physical Higgs boson of the standard GWS model serves to regulate the divergences of the restricted theory, thus its mass provides a cutoff scale for the model studied here.

The problem of calculating quantum corrections in the non-linear $\sigma$ model Las a iong history. The covariant loop expansion for the ungauged model was constructed in the early 70s by Honerkamp[19], using functional methods to construct the expansion of $Z(J)$; however he did not actually calculate explicitly any higher order terms. That problem was tackled later by Appelquist and Bernard, [20] using a non-covariant diagrammatic expansion. They found divergences violating the original glubal symmetry which vanished on shell, but required complicated field redefinitions to restore the symmetry off-shell. Gaillard[10] recently extended the covariant approach of Honerkamp using the methods described in the last chapter, to find the coefficient of the two derivative term at one loop. Appelquist and Bernard also studied the non-linear $\sigma$ model with gauged $\mathrm{SU}(2)_{L}$, and found a counterterm structure equal to the results found 
below in the limit of vanishing Weinberg angle. Finally, Longhitano [17] did a diagrammatic one-loop calculation in the full gauged $S U(2)_{L} \times U(1)$ model, and found the same terms which we compute below.

The GWS model possesses a local symmetcy group $S U(2)_{L} \times U(1)$, and in the minimal version studied here, a larger global symmetry group $\mathrm{SU}(2)_{L} \times \mathrm{SU}(2)_{R}$ is also present at tree level, except for violations due to the $U(1)$ gauge coupling (the third component of $\mathrm{SU}(2)_{R}$ is the global part of the local $U(1)$ symmetry). This "custodial" symmetry leads to the mass relation among the gauge bosons $\rho \equiv \frac{M_{W}^{2}}{M_{Z}^{2} \cos ^{2} \theta}=1$. Violations of the global symmetry by quantum effects give rise to observable physical consequences, such as corrections to this mass relation.

\section{The One Loop Caiculation}

The Lagrangian we study for this model is that of the GWS model without the fermions:

$$
\mathcal{L}=-\frac{1}{2} \operatorname{tr} F_{W}^{2}-\frac{1}{4} F_{B}^{2}+\frac{1}{2}\left|D_{\mu} \Phi\right|^{2}
$$

where the gauge boson field strengths are given by

$$
F_{W_{\mu \nu}}=\frac{i}{g}\left[\partial_{\mu}-i g W_{\mu}, \partial_{\nu}-i g W_{\nu}\right] \quad \text { with } \quad W_{\mu}=W_{\mu}^{a} \frac{\tau_{a}}{2}
$$

(where $\tau_{a}$ are the Pauli matrices), and

$$
F_{B_{\mu}}=\frac{i}{g^{\prime}}\left[\partial_{\mu}-i g^{\prime} B_{\mu}, \partial_{\nu}-i g^{\prime} B_{\nu}\right]
$$

and the covariant derivative on $\Phi$ is

$$
\mathbf{D}_{\mu}=\partial_{\mu}-i g W_{\mu}-\frac{i g^{\prime}}{2} B_{\mu}=\mathbf{D}_{0 \mu}-i g\left(\widetilde{W}_{\mu}+\frac{1}{2} \tan \theta \widetilde{B}_{\mu}\right)
$$

In this last equation, the gauge fields have been split into a background piece, in $\mathbf{D}_{0, \mu}$, and the quantum piece, marked by a tilde. Also, $\tan \theta \equiv \frac{g^{\prime}}{g}$ is the tangent of the Weinberg angle. 
We also need to split the Higgs field into background and quantum pieces, and rather than doing the usual additive splitting, we take $\Phi=V u \Phi_{0}$, where $V$ and $u$ are $\mathrm{SU}(2)$ matrices,

$$
\Phi_{0} \equiv\left(\begin{array}{l}
0 \\
v
\end{array}\right)
$$

is a constant vector, and the background part of $\Phi$ is $V \Phi_{0}$. With these conditions $\Phi$ satisfies the constraint $|\Phi|^{2}=v^{2}$. (Because of the factor of $\frac{1}{2}$ in front of the scalar kinetic energy, the scale parameter $v$ is normalized in the conventional way.) The quantum part of the scalar field is

$$
u \equiv 1+i x \quad \text { with } \quad z=z_{a} \frac{\tau^{a}}{2}+\mathcal{O}\left(z_{a}^{2}\right) .
$$

To proceed, we rotate the background scalar fields into the gauge fields, defining

$$
\overline{\mathbf{D}}_{\mu}=\partial_{\mu}+V^{-1} \mathbf{D}_{0 \mu} V \equiv \partial_{\mu}-i G_{\mu}
$$

and

$$
X_{\mu}=X_{\mu}^{a} \frac{\tau_{a}}{2} \equiv V^{-1} \widetilde{W}_{\mu} V
$$

This looks like we are gauge fixing to unitary gauge for the background fields, but in fact the scalar degrees of freedom are still present, hidden in $G_{\mu}$. There is no background field gauge fixing. The covariant derivative of $\Phi$ may now be expressed as

$$
D_{\mu} \Phi=V\left(\bar{D}_{\mu}-i g\left(X_{\mu}+\frac{1}{2} \tan \theta \widetilde{B}_{\mu}\right)\right)(1+i z) \Phi_{0}
$$

We can now rewrite the kinetic term for the scalars as

$$
\begin{aligned}
\frac{1}{2}\left|D_{\mu} \Phi\right|^{2} & =\frac{1}{2}\left|\bar{D}_{\mu} u \Phi_{0}\right|^{2}+\frac{g v^{2}}{2} \operatorname{tr}\left[z_{1}\left\{\bar{D}_{\mu}, X^{\mu}+\frac{1}{2} \tan \theta \widetilde{B}^{\mu}\right\}\right] P \\
& +\frac{g^{2} v^{2}}{2} \operatorname{tr}\left(X_{\mu}+\frac{1}{2} \tan \theta \widetilde{B}_{\mu}\right)^{2} P \\
& + \text { terms not bilinear in quantum fields, }
\end{aligned}
$$

where $P \equiv \frac{1-\tau_{3}}{2}=\left(\begin{array}{ll}0 & 0 \\ 0 & 1\end{array}\right)$. The first term is the kinetic operator for the scalars covariant only with respect to background field gauge transformations. 
We will come back to it in a moment. The second term mixes the quantum scalar and gauge fields. It may be rewritten as

$$
\mathcal{L}_{G-x}=\frac{g v^{2}}{2} \operatorname{tr} x\left(\left[\bar{D}_{\mu},\left\{X^{\mu}+\frac{1}{2} \tan \theta \bar{g}^{\mu}, P\right\}\right]+i\left[\left\{P, G_{\mu}\right\}_{,} X^{\mu}\right\}\right)
$$

The components of the commutator of $\bar{D}$ with a matrix $A$ may be written as

$$
\left(\left[\overline{\mathbf{D}}_{\mu}, A\right]\right)^{i}=d_{\mu}^{i j} A_{j} \quad \text { where } \quad d_{\mu}^{i j}=\partial_{\mu} \delta^{i j}-\epsilon_{k}^{i j} G_{\mu}^{k}, \quad(i, j=0, \ldots, 3),
$$

and $\epsilon^{i j}{ }_{k}$ is defined to vanish if its indices are not a permutation of $\{1,2,3\}$. The commutator with $\overline{\mathbf{D}}$ generates a piece which is not in the $\mathrm{SU}(2)$ algebra; however in the present case this piece may be neglected, because the part of $z$ not in the $\mathrm{SU}(2)$ algebra gives rise to a term which is $3^{\text {rd }}$ order in quantum fieldis (equation (3.6)). With this in mind we may rewrite the cross term as

$$
\mathcal{L}_{G-1}=\frac{g v^{2}}{4} z_{i}\left(d_{\mu} K Y^{\mu}+i \widehat{G}_{\mu} Y^{\mu}\right)^{i}
$$

where $Y^{\mu}$ is a 4 component gauge field defined by

$$
\begin{aligned}
& Y_{a}^{\mu}=X_{a}^{\mu} \quad \text { for } \quad a=1,2,3 \\
& Y_{0}^{\mu}=B^{\mu}
\end{aligned}
$$

and $K$ and $\widehat{G}_{\mu}$ are given by

$$
K=\left(\begin{array}{cccc}
1 & 0 & 0 & \tan \theta \\
0 & 1 & 0 & 0 \\
0 & 0 & 1 & 0 \\
-\tan \theta & 0 & 0 & 1
\end{array}\right) \quad \text { and } \quad \hat{G}_{\mu}^{i j}=-i \epsilon_{k}^{i j} \hat{G}_{\mu}^{k}=-i \epsilon_{k}^{i j}\left(G_{\mu}^{k}-\delta^{k J} G_{\mu}^{0}\right)
$$

Note that $K Y$ is not exactly the same as the anticommutator of $P$ with the gauge field. We have

$$
\left(K Y_{\mu}\right)_{a}=\left\{P, X_{\mu}+\frac{1}{2} \tan \theta \tilde{B}_{\mu}\right\}_{a} \quad \text { for } \quad a=1,2,3
$$

but 


$$
\left(K \Psi_{\mu}\right)_{0} \neq\left\{P, X_{\mu}+\frac{1}{2} \tan \theta \widetilde{B}_{\mu}\right\}_{0}
$$

However, the remark made above equation (3.12) tells us that the 0 component doesn't matter here. This choice for $K$ is convenient because it allows us to include the gauge condition for the photon in a natural way, along with the gauge condition for the $W \mathbf{s}\left(\left(K Y_{\mu}\right)_{0}\right.$ is proportional to the photon field). To gauge fix, we impose the background-field Landau gauge condition, setting $d_{\mu} Y^{\mu}=0$. Applying this condition to the gauge-scalar mixing term leaves

$$
\mathcal{L}_{G-s}=\frac{g v^{2}}{4} x \mathcal{J}_{\mu} Y^{\mu} \quad \text { where } \quad \mathcal{J}_{\mu} \equiv\left[d_{\mu}, K\right]+i \widehat{G}_{\mu}
$$

The last term in equation (3.9) gives the gauge bosons their mass, and may be rewritten as

$$
\frac{g^{2} v^{2}}{8} Y_{\mu} K^{T} N K Y^{\mu} \equiv \frac{1}{2} Y_{\mu} M_{G}^{2} Y^{\mu}
$$

where $N=\operatorname{diag}(0,1,1,1)$, by observing that for any matrix $A$ we have

$$
\operatorname{tr} A^{2} P=\frac{1}{4}\left[\operatorname{tr}\{P, A\} \tau_{a}\right]^{2}=\frac{1}{4} \sum_{a=1}^{3}\left[\{P, A\}_{a}\right]^{2}
$$

and using equation (3.15).

Returning to the first term, we have the kinetic operator for the physical Higgs and the Goldstone modes, subject to the constraint that the physical Higgs field be fixed at its ground state value. It is easiest to work here with "Cartesian" coordinates $\left(\sigma, \pi_{a}\right)$ for the quanturn scalar field, with $u=\frac{\sigma+i \pi \cdot T}{v}$ (which gives $v z_{a}=2 \pi_{a}+\mathcal{O}\left(\pi^{2}\right)$ ). There are only three free components, and we may choose $\sigma$ to be constrained: $\sigma^{2}=v^{2}-\pi^{2}$. After a bit of algebra, we find for the scalar kinetic term

$$
\frac{1}{2}\left|\overline{\mathbf{D}}_{\mu} \Phi\right|^{2}=-\frac{1}{2} \pi_{a}\left(\mathcal{D}^{2}+M_{\pi}^{2}\right)^{a b} \pi_{b} \equiv-\frac{1}{2} \pi_{a} \Delta_{\pi}^{-1} \pi_{b},
$$

where we have added a total divergence, and defined

$$
\mathcal{D}_{\mu}^{a b}=\partial_{\mu} \delta^{a b}-\frac{1}{2} \epsilon^{a b}{ }_{c}\left(G_{\mu}^{c}+\delta^{3 c} G_{\mu}^{0}\right)=d_{\mu}^{a b}+\frac{i}{2} \hat{G}_{\mu}^{a b}, \quad(a, b=1,2,3),
$$


and

$$
M_{\pi}^{2 a b}=\frac{1}{4}\left(\widehat{G}_{\mu} \widehat{G}^{\mu}\right)^{a b} .
$$

The gauge kinetic energy terms may be rewritten in terms of $Y$ and the field strength $\bar{F}_{W}$ given by

$$
\bar{F}_{W \mu \nu} \equiv \frac{i}{g} V^{-1}\left[\partial_{\mu}-i g W_{0_{\mu}}, \partial_{\nu}-i g W_{0_{\nu}}\right] V,
$$

where $W_{0, \mu}$ is the background part of $\boldsymbol{W}$. In terms of components we have

$$
\bar{F}_{W \mu \nu}^{a b}=\frac{i}{g}\left(\left[d_{\mu}, d_{\nu}\right]\right)^{a b}, \quad \bar{F}_{W \mu}^{0 i}=\bar{F}_{W \mu \nu}^{i 0}=0 .
$$

Including only terms bilinear in the quantum field $Y$ gives for the relevant part of the gauge kinetic energy,

$$
\mathcal{L}_{G}=-\frac{1}{2} \text { tr } F_{W}^{2}-\frac{1}{4} F_{B}^{2} \rightarrow \frac{1}{2} Y_{\mu}\left(d^{2} g^{\mu \nu}-d^{\mu} d^{\nu}-2 i g \bar{F}_{W}^{\mu \nu}\right) Y_{\nu}
$$

Adding to this the gauge mass term from the scalar kinetic energy gives

$$
\mathcal{L}_{G}=\frac{1}{2} Y_{\mu}\left(d^{2} g^{\mu \nu}-d^{\mu} d^{\nu}-2 i g \bar{F}_{W}^{\mu \nu}+M_{G}^{2} g^{\mu \nu}\right) Y_{\nu} .
$$

We may simplify the gauge kiuetic operator as in the QCD calculation: applying the gauge fixing constraint $d_{\mu} Y^{\mu}=0$ leaves us with

$$
\mathcal{L}_{G}=\frac{1}{2} Y_{\mu}\left(d^{2} g^{\mu \nu}-2 i g \bar{F}_{W}^{\mu \mu}+M_{G}^{2} g^{\mu \nu}\right) Y_{\nu} \equiv \frac{1}{2} Y_{\mu} \Delta_{G}^{-1 / \omega} Y_{\nu} .
$$

In order to impose the gauge condition we introduce an auxiliary field, as before. Then the part of the Lagrangian relevant to the one loop calculation is

$$
\mathcal{L}=\frac{1}{2} Y_{\mu} \Delta_{G}^{-1 / \omega} Y_{\nu}-\frac{1}{2} \pi \Delta_{\pi}^{-2} \pi-i \alpha d_{\mu} Y^{\mu}+\frac{1}{2} g v \pi J_{\mu} Y^{\mu}
$$

In order to eliminate the $Y-\pi$ and $Y-\alpha$ cross terms, we shift $Y$. This gives a $\pi-\alpha$ cross term, which can also be shifted away. The result of all this is a Lagrangian of the form

$$
\begin{aligned}
\mathcal{L} & =\frac{1}{2} Y_{\mu} \Delta_{G}^{-1 / \omega} Y_{\nu}-\frac{1}{2} \alpha d_{\mu} \Delta_{G}^{\prime \mu} d_{\nu} \alpha \\
& -\frac{1}{2} \pi_{a}\left[\Delta_{\pi}^{-1}+\frac{g^{2} v^{2}}{4}\left(\mathcal{J}_{\mu} \Delta_{G}^{\prime \mu \nu} \mathcal{J}_{\nu}^{\gamma}-\left(\mathcal{J}_{\mu} \Delta_{G}^{\mu \nu} d_{\nu}\right)\left(d_{\rho} \Delta_{G}^{\mu \sigma} d_{\sigma}\right)^{-1}\left(d_{\lambda} \Delta_{G}^{\lambda_{k}} \mathcal{J}_{\kappa}^{T^{2}}\right)\right)\right]^{\alpha b} \pi_{b} .
\end{aligned}
$$


Note that the indices in the $\pi$ term run only from 1 to 3 (not 0 to 3). Finally, then, the one loop effective action for this model is given by

$$
S_{1}=\frac{i}{2} \operatorname{Tr} \ln \Delta_{G}^{-1 \mu \nu}-i \operatorname{Tr} \ln d^{2}+\frac{i}{2} \operatorname{Tr} \ln d_{\mu} \Delta_{G}^{i \mu} d_{\nu}+\frac{i}{2} \operatorname{Tr} \ln (\pi \operatorname{term}) .
$$

The careful reader may be wondering what has happened to the background field gauge invariance after all these manipulations. The only possible source of difficulty is the $\mathrm{U}(1)$ transformation $-G_{\mu}$ and things built from from it are invariant under the background $S U(2)$. Under the $U(1)$, on the other hand, $G_{\mu}$ is not invariant. This is a good thing, because it appears as the gauge field in a covariant derivative. The background field covariant derivative transforms as

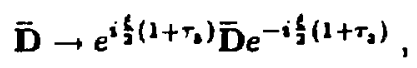

with gauge function $\xi$. From this we find that the infinitesimal cliange in $\widehat{G}$ is given by $\delta \widehat{G}_{\mu}^{a}=\xi \epsilon_{b}^{3 a} \widehat{G}_{\mu}^{b}$, i.e., $\widehat{G}_{\mu}$ transforms homogeneously, and $\widehat{G}_{\mu}^{3}$ is an invariant. This is what we should expect: the components of $\hat{G}_{\mu}^{a}$ are proportional to the physical $W^{ \pm}$and $Z$ fields, and the remnant of the original $\operatorname{SU}(2) \times U(1)$ is the electromagnetic $U(1)$ which just transforms $W_{1}$ and $W_{2}$ into each other. In the $4 \times 4$ basis for the fields and operators the transformation is generated by

$$
\Omega=\xi\left(\begin{array}{cccc}
0 & 0 & 0 & 0 \\
0 & 0 & 1 & 0 \\
0 & -1 & 0 & 0 \\
0 & 0 & 0 & 0
\end{array}\right)
$$

Since both $K$ and $M_{G}^{2}$ commute with $\Omega$, we also find that $\mathcal{J}_{\mu}$ and commutators involving $M_{G}^{2}$ transform honugeneously. We may conclude, then, that things that look like invariants constructed from these objects (that is, things with all indices contracted), really are invariants.

Except for the presence of the mass term, the gauge loop contribution is of the form already calculated in section 3 of the last chapter. In fact, though, the addition of the mass term has no effect on the field dependent divergences at 
one loop. Following the derivation of equations (2.54) and (2.59) we find for the one loop divergent terms, dimensionally regulated,

$$
\frac{i}{2} \operatorname{Tr} \ln \left(d^{2}+M_{G}^{2}\right)=\frac{1}{\epsilon} \cdot \frac{1}{64 \pi^{2}} \int d x\left(\operatorname{tr} M_{G}^{4}-\frac{g^{2}}{6} \operatorname{tr} \bar{F}_{W, W} \bar{F}_{W}^{\prime w}\right)+\text { finile }
$$

If we regulate inslead with a double subtraction at mass scale $\Lambda[10]$, we find also a quadratically divergent part

$$
-\frac{\Lambda^{2}}{32 \pi^{2}} \operatorname{tr} M_{G}^{2}
$$

(We know that $\Lambda$ is proportional to the Higgs mass $M_{H}$, but in this approach there's no way to identify the constant of proportionality, as it depends on the details of the symmetry breaking physics). For the gauge contribution, though, since $M_{G}^{2}$ is field-independent the only non-trivial contribution is the field-strength term which requires a wave-function renormalization for the $W$. This term is exactly the same as the result from the calculation in section 3 above, after the ghost is included:

$$
\begin{aligned}
& \frac{1}{\epsilon} \cdot \frac{1}{16 \pi^{2}} \cdot \frac{11}{12} g^{2} \operatorname{tr} F_{W \mu} F_{W}^{\prime \omega} \\
= & \frac{1}{\epsilon} \cdot \frac{1}{16 \pi^{2}} \cdot \frac{11}{3} g^{2} \operatorname{tr} F_{W_{W}} F_{W}^{\prime \omega} .
\end{aligned}
$$

(We are being somewhat cavalier about the dimension of these terms in dimensional regularization; to find the renormalization group equations one must be more careful, as in the QCD calculation above. Also, we have dropped the bar over the field strength: all fields appearing in the one loop effective Lagrangian are background fields.)

In the QCD calculation we found that the auxiliary field term vanished identically. For the present case, it turns out that the auxiliary term again makes no contribution to the divergent terms. The auxiliary term may be considerably simplified by manipulating tine identity

$$
1=\frac{1}{d^{2}} d^{\rho} \Delta_{G}^{-1}{ }_{p \mu}^{\mu} \Delta_{G}^{\mu \nu} d_{\nu},
$$


to give

$$
d_{\mu} \Delta_{G}^{\mu \nu} d_{\nu}=1-\frac{1}{d^{2}+M_{G}^{2}}\left[M_{G}^{2}+\left(\left[d_{\mu}, M_{G}^{2}+i g\left[d^{\rho}, \bar{F}_{W \mu \rho}\right]\right) \Delta_{G}^{\mu \nu} d_{\nu}\right] .\right.
$$

Then, expanding the logarithm (equation (3.28)), and retaining only terms which will give a divergent part, the auxiliary field term becomes

$$
\begin{aligned}
& \frac{i}{2} \operatorname{Tr} \ln d_{\mu} \Delta_{G}^{\mu \nu} d_{\nu}= \\
& -\frac{i}{2} \operatorname{Tr}\left[\frac{1}{d^{2}+M_{G}^{2}}\left(M_{G}^{2}+\left(\left[d_{\mu}, M_{G}^{2}\right]+i g\left[d^{\rho}, \bar{F}_{W \mu \rho}\right]\right) \frac{1}{d^{2}+M_{G}^{2}} d^{\mu}\right)\right]+\text { finite. }
\end{aligned}
$$

The first term inside parentheses gives rise to a field independent divergent part. The remaining terms also leave only finite parts: the log divergent part of the field strength term vanishes on taking the trace (and using the jacobi identity), and the divergent part of the other term is proportional to $\operatorname{tr}\left[d_{\mu, 1}\left[d_{\nu,}, M_{G}^{2}\right]\right]$, which vanishes on integration by parts in the effective action.

Finally, we have the scalar term, which looks more intimidating than it really is. We can split it into two pieces:

$$
\begin{aligned}
S_{\pi} & =\frac{i}{2} \operatorname{Tr} \ln \Delta_{\pi}^{-1} \\
& +\frac{i}{2} \operatorname{Tr} \ln \left[1+\frac{g^{2} v^{2}}{4} \Delta_{\pi}\left(\mathcal{J}_{\mu} \Delta_{G}^{\prime \mu} \mathcal{J}_{\nu}^{T}-\left(\mathcal{J}_{\mu} \Delta_{G}^{\mu \nu} d_{\nu}\right)\left(d_{\rho} \Delta_{G}^{\rho \sigma} d_{\sigma}\right)^{-1}\left(d_{\lambda} \Delta_{G}^{\lambda \kappa} \mathcal{J}_{\kappa}^{T}\right)\right)\right] .
\end{aligned}
$$

The first piece is of exactly the same form as equation (3.31), except that in this case the mass term is a function of the background fields, so that the quadratic divergence which was discarded in the gauge loop calculation cannot be dropped here. It's value is

$$
-\frac{\Lambda^{2}}{32 \pi^{2}} \operatorname{Tr} M_{\pi}^{2}=-\frac{\Lambda^{2}}{64 \pi^{2}} \widehat{G}_{12}^{a} \widehat{G}_{a}^{\prime \prime} .
$$

Some useful relations for re-expressing terms involving $\widehat{G}_{\mu}$ as terms involving $\Phi$ are

$$
\widehat{G}_{\mu}=\left\{P, G_{\mu}\right\}-\operatorname{tr} P G_{\mu}, \quad \operatorname{tr} P X=\frac{1}{v^{2}} \Phi_{0}^{\prime} X \Phi_{0}
$$

and 


$$
\text { tr } P X P Y=\operatorname{tr} P X \operatorname{tr} P Y \text {. }
$$

Then (3.38) may be rewritten in terms of the background scalar and gauge fields as

$$
-\frac{1}{16 \pi^{2}} \frac{\Lambda^{2}}{v^{2}}\left|D_{\mu} \Phi\right|^{2}
$$

where $D_{\mu}$ and $\Phi$ now include only the background fields. This term thus represents a quadratically divergent scalar wave-function renormalization (of course, it vanishes in dimensional regularization). Such a term would be very surprising in the linear theory from which this one was derived (in fact, it would be wrong); here it arises because of extra derivative couplings induced by taking the non-linear limit for the Lagrangian. This is the only quadratic divergence in the one loop approximation. Since it has exactly the same form as the scalar kinetic term in the original Lagrangian it does not affect the physics-it can be absorbed by a redefinition of $v$. Hence we have Veltman's "screening theorem" [13]: there are no physical one-loop effects quadratic in $\boldsymbol{M}_{\boldsymbol{H}}$.

The logarithmically divergent terms arising from the first trace in equation (3.37) are given by (as in equation (3.31))

$$
\frac{1}{\epsilon} \cdot \frac{1}{64 \pi^{2}}\left(\operatorname{tr} M_{\pi}^{4}-\frac{1}{6} \operatorname{tr} \Psi_{\mu \nu} \Psi^{\mu \nu}\right) \quad \text { where } \quad \Psi_{\mu \nu} \equiv i\left[\mathcal{D}_{\mu}, \mathcal{D}_{\nu}\right]
$$

To analyze these terms, we switch back from $3 \times 3$ matrix notation to the original $2 \times 2$ notation. In this notation, the terms in (3.41) can be rewritten as

$$
\operatorname{tr} M_{\pi}^{4}=\frac{1}{4}\left[\operatorname{tr} \widehat{G}_{\mu} \widehat{G}^{\mu}\right]^{2}+\frac{1}{4}\left[\operatorname{tr} \widehat{G}_{\mu} \widehat{G}_{\nu}\right]^{2} \quad \text { where } \quad \widehat{G}_{\mu}=G_{\mu}^{a} \frac{\tau_{a}}{2}-G_{\mu}^{0} \frac{\tau_{3}}{2},
$$

and

$$
\operatorname{tr} \Psi_{\mu \nu}^{2}=\operatorname{tr}\left(g V^{-1} F_{W \mu} V+\frac{1}{2} g^{\prime} F_{B_{\mu}} \tau_{3}+\frac{i}{2}\left[\hat{G}_{\mu,} \hat{G}_{\nu}\right]\right)^{2}
$$

The contribution of these terms can now be expressed as

$$
\frac{1}{\epsilon} \cdot \frac{1}{64 \pi^{2}}\left[-\frac{g^{2}}{6} \operatorname{tr} F_{W}^{2}-\frac{g^{\prime 2}}{12} F_{B}^{2}-\frac{g g^{\prime}}{6} F_{B_{\mu \nu}} \operatorname{tr} \tau_{3} V^{-1} F_{W}^{\prime \mu} V\right.
$$




$$
\begin{aligned}
-\frac{i}{6} \operatorname{tr}\left(g V^{-1} F_{W_{L}} V\right. & \left.+\frac{g^{\prime}}{2} F_{B_{\mu} \tau_{3}}\right)\left[\widehat{G}^{\mu}, \widehat{G}^{\nu}\right] \\
& \left.+\frac{1}{6}\left(t \widehat{G}_{\mu}^{2}\right)^{2}+\frac{1}{3}\left(\operatorname{tr} \widehat{G}_{\mu} \widehat{G}_{\nu}\right)^{2}\right] .
\end{aligned}
$$

It is a simple matter to find the divergent part of the remaining trace in equation (3.37). That divergence is die to the leading term in the expansion of the logarithm, and can be computed simply by substituting

$$
\Delta \rightarrow \frac{1}{-p^{2}+m^{2}}
$$

where the mass here is arbitrary; the corrections to this substitution give convergent integrals. Thus we obtain from this term a contribution

$$
-\frac{1}{\epsilon} \cdot \frac{3 g^{2} v^{2}}{512 \pi^{2}} \operatorname{tr} \mathcal{J}^{\mu} \mathcal{J}_{\mu}^{T}
$$

The trace is only over index values from 1 to 3 , because the $\pi$ field has no 0 component. After evaluating the trace, (3.45) becomes

$$
-\frac{1}{\epsilon} \cdot \frac{3 g^{2} v^{2}}{512 \pi^{2}}\left[\left(2+\tan ^{2} \theta\right) \hat{G}^{2}-\tan ^{2} \theta\left(\hat{G}_{3}\right)^{2}\right]
$$

which can be rewritten as

$$
-\frac{1}{\epsilon} \cdot \frac{3 g^{2}}{128 \pi^{2}}\left[\left(2+\tan ^{2} \theta\right)\left|\mathbf{D}_{\mu} \Phi\right|^{2}+\frac{1}{v^{2}} \tan ^{2} \theta\left(\Phi^{\dagger} \mathbf{D}_{\mu} \Phi\right)^{2}\right]
$$

We now have the complete one loop calculation of heavy Higgs dependent terms. Adding up the pieces from (3.33), (3.40), (3.44), and (3.47) we find for the unrenormalized one loop effective Lagrangian

$$
\begin{aligned}
\mathcal{L}_{1}= & \mathcal{L}-\frac{1}{16 \pi^{2}} \frac{\Lambda^{2}}{v^{2}}\left|D_{\mu} \Phi\right|^{2}+\frac{1}{\epsilon} \cdot \frac{1}{16 \pi^{2}}\left[\frac{29 g^{2}}{8} \operatorname{tr} F_{W}^{2}-\frac{g^{\prime 2}}{48} F_{B}^{2}\right. \\
& -\frac{g g^{\prime}}{24} F_{B \mu} \operatorname{tr} \tau_{3} V^{-1} F_{W}^{\mu \nu} V-\frac{i}{24} \operatorname{tr}\left(g V^{-1} F_{W, \mu} V+\frac{g^{\prime}}{2} F_{B, \mu} \tau_{3}\right)\left[\widehat{G}^{\mu}, \widehat{G}^{\nu}\right] \\
& -\frac{3 g^{2}}{8}\left(2+\tan ^{2} \theta\right)\left|D_{\mu} \Phi\right|^{2}-\frac{3 g^{2} \tan ^{2} \theta}{8 v^{2}}\left(\Phi^{\prime} D_{\mu} \Phi\right)^{2} \\
& \left.+\frac{1}{24}\left(\operatorname{tr} \widehat{G}_{\mu}^{2}\right)^{2}+\frac{1}{12}\left(\operatorname{tr} \widehat{G}_{\mu} \widehat{G}_{\nu}\right)^{2}\right] .
\end{aligned}
$$


These terms (except for those on the first line) were previously found by Longhitano [17] using diagrammatic methods. The terms in this expression are given in a mixed form: those of the same form as terms in the original Lagrangian or which give a new gauge boson mass term have been written so that the correspondence is apparent. Those which generate new 3-point or 4-point vertices for the gauge bosons have been written so that the coefficients of thuse vertices can be extracted easily.

\section{Phenomenological Consequences}

The divergences in equation (3.48) which correspond to terms in the original Lagrangian are genuine, in the sense that they are not regulated by calculating in the full linear theory with a propagating physical Higgs. The other divergences are cut off in the linear theory. By comparing the dimensional regularization with a cutoff or subtraction regularization we may then conclude that the $\boldsymbol{M}_{H}$ dependence of these terms in the linear theory is given by the replacement [20] $\frac{1}{\epsilon} \rightarrow \ln \frac{M_{H}^{2}}{M_{W}^{2}}$. The reason for putting $M_{W}^{2}$ in this expression to set the scale is that the terms whose divergence is cut off in the linear theory are (in this gauge) just those arising from the scalar determinant. By looking at the theory in unitary gauge we know that the physical modes propagate with mass $M_{W}$ (or $M_{Z}$, which is the same to this order), and this scale therefore cuts off the momentum integrals at small momenta. The presence of massless photons is irrelevant to the terms with a physical dependence on $M_{H}$ because the gaugs: boson loops give rise only to fully renormalizable divergences. The terms found here are the only ones which grow like $\ln M_{H}^{2}$ at one loop, even in the full theory including fermions. This is because the fermion sector of the thenry is renormalizable even in the limit of large Higgs mass. The extra infinities induced by the nonrenormalizability of the scalar sector thus enter the fermion sector only at two loops, and it is only these latter infinities which are cut off by the renormalizable linear theory to leave a pliysical dependence on the Higgs 
mass.

We can casily extract the correction to $\rho$ induced by these one-loop terms. A definition of $\rho$ beyond tree level which serves well is as the ratio of neutral current to charged current scattering amplitudes [17] which depend on $M_{Z}^{2} \cos ^{2} \theta$ and $M_{W}^{2}$, or equivalently, through neutral current scattering and the muon $d_{-2}$ cay constant $G_{F}[15]$. These quantities are determined by diagrams which irvolve only internal gauge boson lines. At small $q^{2}$ (invariant mass) the gauge bosons' wave function renormalization cancels between the vertex factors and the vector propagator, so for $\rho$ defined in this way we may ignore wave function renormalization. Then $\rho$ is determined entirely by the effective mass term

$$
-\frac{3 g^{2}}{128 \pi^{2} v^{2}} \tan ^{2} \theta\left(\Phi^{1} D_{\mu} \Phi\right)^{2} \ln \frac{M_{H}^{2}}{M_{W}^{2}}
$$

(The renormalization of the scalar kinetic energy $\frac{1}{2}\left|D_{\mu} \Phi\right|^{2}$ in the original Lagrangian doesn't affert this calculation-it rescales the $W$ and $Z$ masses by the same factor.) This term represents a mass shift for the $Z$, as can easily be seen in unitary gauge:

$$
\left(\Phi^{\dagger} \mathbf{D}_{\mu} \Phi\right)^{2} \rightarrow-\frac{g^{2} v^{4}}{4 \cos ^{2} \theta} Z^{2}
$$

where the normalized $Z$ field is given by $Z_{\mu}=\cos \theta\left(W_{3 \mu}-\tan \theta B_{\mu}\right)$. Plugging this in to (3.49) gives

$$
\frac{3 g^{2}}{64 \pi^{2}} \tan ^{2} \theta\left(\frac{g^{2} v^{2}}{8 \cos ^{2} \theta} Z^{2}\right) \ln \frac{M_{H}^{2}}{M_{W}^{2}}
$$

The factor in parentheses is the $Z$ mass term from the original Lagrangian, thus we obtain a correction to the $\rho$ parameter of

$$
\rho=1-\frac{3 g^{2}}{64 \pi^{2}} \tan ^{2} \theta \ln \frac{M_{H}^{2}}{M_{W}^{2}},
$$

The correction to $\rho$ found here is in agreement with previous calculations by diagrammatic methods[15,17]. It is interesting to see that this correction could be computed from a single term in the derivative expansion. Unfortunately, this 
result affords little opportunity for extracting the Higgs mass from low energy measurements: for a Higgs mass of $1 \mathrm{TeV}$, equation (3.52) gives a shift in $\rho$ of only .003. Other measurements may afford a more sensitive probe [18].

We may also use the last two terms to estimate the corrections to the low energy amplitudes for $W$ and $Z$ scattering found by Chanowitz and Gaillard [21], who showed that the amplitude for the scattering of longitudinal gauge bosons at center of mass energies $M_{W} \ll \sqrt{s} \ll M_{H}$ is equal to the appropriate amplitude for scattering of the Goldstone scalars $\left(\pi_{a}\right)$, up to corrections of order $\frac{M_{W}}{\sqrt{s}}$. (Similar, though less involved calculations were done earlier by Lee, Quigg and Thacker for the case $\sqrt{s} \gg M_{H}[22]$.) Using this approach to find, say, the amplitude for the scattering of longitudinal $W \mathrm{~s}, W_{L}^{+} W_{L}^{-} \rightarrow W_{L}^{+} W_{L}^{-}$, we obtain at tree level the amplitude $[21,22]$

$$
A_{v}\left(W_{L}^{+} W_{L}^{-} \rightarrow W_{L}^{+} W_{L}^{-}\right)=-\frac{1}{v^{2}} u
$$

where $u$ is the square of the difference between the incoming $\mathrm{W}^{+}$and outgoing $W^{-}$momenta. We can obtain the one loop corrections to this amplitude by rewriting the last two terms from the effective Lagrangian (3.48) in terms of the scalars, and dropping the gauge fields. We find for the new effective interaction among the scalars the expression

$$
\mathcal{L}_{\text {uculars }}=\frac{1}{96 \pi^{2} v^{4}} \ln \frac{M_{H}^{2}}{M_{W}^{2}}\left[2\left(\eta^{a b} \partial_{\mu} \pi_{a} \partial_{\nu} \pi_{b}\right)^{2}+\left(\eta^{a b} \partial_{\mu} \pi_{a} \partial^{\mu} \pi_{\iota}\right)^{2}\right]
$$

where

$$
\eta^{a b}=\delta^{a b}+\frac{\pi^{a} \pi^{b}}{v^{2}-\pi^{2}}
$$

is the metric for the Goldstone scalars on the internal symmetry manifold. From this we obtain for the contribution to the scattering amplitude from the one loop correction

$$
A_{1}\left(W_{L}^{+} W_{L}^{-} \rightarrow W_{L}^{+} W_{L}^{-}\right)=\frac{1}{24 \pi^{2} v^{4}} \ln \frac{M_{H}^{2}}{M_{W}^{2}}\left(s^{2}+t^{2}+u^{2}\right)
$$


where $s$ is the square of the center of mass energy and $t$ is the square of the difference between incoming and outgoing momenta of the $W^{+}$. A similar calculation gives for the combined tree and one loop contributions to the other neutral scattering processes

$$
A\left(Z_{L} Z_{L} \rightarrow Z_{L} Z_{L}\right)=\frac{1}{16 \pi^{2} v^{4}} \ln \frac{M_{H}^{2}}{M_{W}^{2}}\left(s^{2}+t^{2}+u^{2}\right),
$$

and

$$
A\left(W_{L}^{+} W_{L}^{-} \rightarrow Z_{L} Z_{L}\right)=\frac{s}{v^{2}}+\frac{1}{48 \pi^{2} v^{4}} \ln \frac{M_{H}^{2}}{M_{W}^{2}}\left(s^{2}+t^{2}+u^{2}\right)
$$

Interestingly, the $Z Z \rightarrow Z Z$ channel has no tree contribution, and a relatively large one loop contribution, which turns out to be comparable to the tree amplitudes in the other channels at energies of $\sqrt{s} \sim 1 \mathrm{TeV}$, for a Higgs mass of order $1 \mathrm{TeV}$.

It should be pointed out here that the applicability of the loop expansion in this model is not completely obvious. If $M_{H}$ is sufficiently large $\left(M_{H}>1 \mathrm{TeV}\right)$ the model has strong interactions in the scalar sector, and perturbation theory breaks down [22]. Furthermore, it has also been pointed out that the approximation of the full theory by the gauged non-linear $\sigma$ model is invalid beyond one loop [15]. To make higher loop calculations we must sperify the physical details of the high energy cutoff mechanism-the as yet unknown symmetry breaking physics. Of course, higher loop terms will affect these results significantly only if the theory is strongly coupled, so for $M_{H} \lesssim 1 \mathrm{TeV}$ we may take equation (3.48) to be a fairly reliable guide to the size of effects which depend on the symmetry breaking scale, and as indicative of where to find those effects. 


\section{Conclusions}

We have found that it is possible to compute derivative terms in the eflective action from the one loop functional determinant. We have ev.luated the four derivative terms for the theory of a single scalar field with renormalizable self couplings and the two derivative terms for $\mathrm{O}(\mathrm{N})$ symmetric scalar field theory. In these expansions the effective mass of the quantum field as a function of the background field plays the role of an infrared regulator in the loop integral. (The issue of what happens if this effective mass vanishes has been studied by Aitchison and Fraser [8]. They found that the infrared divergences invalidate the derivative expansion, even though the Green functions of the thisory are perfectly well defined, and have sensible zero momentum limits. This problem does not arise in any of the cases studied here, though it would if we sought higher derivative terms in the QCD expansion of chapter 2, or attempted to take the non-linear limit of the $O(N)$ model.)

We have also studied the covariant derivative expansion of the effective action, and seen that it gives a very direct and simple means of evaluating quantities whose diagrammatic calculation may be quite tedious. As examples, we have obtained the one loop $\beta$ function for a Yang-Mills theory with a simple group, and studied the divergences of the gauged non-linear $\sigma$ model. In this last calculation we have found all the terms in the effective action which grow with $M_{H}$ at one loop in the GWS model, interpreting $M_{H}$ as the high energy scale at which the new pliysics responsible for electro-weak symmetry breaking sets in to cul of the divergences of the non-renormalizable low energy theory. We have used these results to find the shift in $\rho$ due to a heavy Higgs, and found surprisingly large one loop contributions to the scattering of longitudinal gauge 
bosons at higlt energy.

Obviously we have only expiored a few of the many potential uses of this expansion. Applications of this method to supergravity and pion plyysics have been studied by Gaillard [10] and by Chan [12]. Any problem of computation in a theory with internal symmetries is a likely candidate for simplification with this method. 


\section{References}

[1] L.F. Abbott, Nuclear Physics B185:189 (1981).

[2] R. Jackiw, Physical Review Do:1686 (1974).

[3] S. Coleman and E. Weinberg, Physical Review D7:1888 (1973).

[4] R. MacKenzie, F. Wilczek and A. Zee, Physical Review Letters 53:2203 (1984), and

R. MacKenzie and F. Wilczek, Physical Review Ds0:2194 (1984).

[5] J. Iliopoulos, C. Itzykson and A. Martin, Reviews of Modern Phy-ics 47:165 (1975).

[6] L.-H. Chan, Physical Review Letters 54:1222 (1985) and Physical Review Letters 55:21 (1985).

[7] C.M. Fraser, Zeitschrift fur Physik 2828:101 (1985).

[8] I.J.R. Aitchison and C.M. Fraser, Physics Letters B146:63 (1984), Physical Review D31:2605 (1985) and Physical Review D32:2190 (1985).

[9] J.A. Zuk, Zeitschrift fur Physik 2829:303 (1985) and Physical Review D32:2653 (1985).

[10] M.K. Gaillard, Nuclear Physics B268:669 (1986).

[11] O. Cheyette, Physical Review Letters 55:2394 (1985).

[12] L.-H. Chan, Physical Review Letters 57:1199 (1986) and MIT Theory preprint MIT-CTP-1390 (1986).

[13] M. Veltman, Acta Physica Polonica B8:475 (1977) and Nuclear Physics B123:89 (1977).

[14] M. Veltman, Physics Letters B01:95 (1980).

[15] J. Van Der Bij and M. Veltman, Nuclear Physics B231:205 (1984). 
[16] M.B. Einhorn, D.R.T. Jones and M. Veitman, Nuclear Plyysics B191:146 (1981).

[17] A.C. Longhitano, Physical Review D22:1166 (1980) and Nuclear Physics B188:118 (1981).

[18] B. Lynn, M. Peskin and R.G. Stuart, Proceedings of the LEP Physics Workshop, CERN Report (1985).

[19] J. Honerkamp, Nuclear Physics B96:130 (1971).

[20] T. Appelquist and C. Bernard, Physical Review D22:200 (1980) and Physical Review D23:425 (1981).

[21] M.S. Chanowitz and M.K. Gaillard, Nuclear Physics B261:379 (1985).

[22] B.W. Lee, C. Quigg and H. Thacker, Physical Review D16:1519 (1977). 